$\mathrm{ROM} 2 \mathrm{~F} / 2008 / 18$

\title{
The Massless Supersymmetric Ladder with $L$ Rungs
}

\author{
G. C. Rossi ${ }^{\dagger}$ and Ya. S. Stanev ${ }^{\ddagger}$ \\ Dipartimento di Fisica, Università di Roma "Tor Vergata" \\ I.N.F.N. - Sezione di Roma "Tor Vergata" \\ Via della Ricerca Scientifica, 1 \\ 00133 Roma, ITALY
}

\begin{abstract}
We show that in the massless $N=1$ supersymmetric Wess-Zumino theory it is possible to devise a computational strategy by which the $x$-space calculation of the ladder 4-point correlators can be carried out without introducing any regularization. As an application we derive a representation valid at all loop orders in terms of conformal invariant integrals. We obtain an explicit expression of the 3-loop ladder diagram for collinear external points.
\end{abstract}

† Giancarlo.Rossi@roma2.infn.it

¥ Yassen.Stanev@roma2.infn.it 


\section{Introduction}

Supersymmetry has many remarkable properties. One of them is that in general supersymmetric theories are less divergent than non-supersymmetric ones [1, 2, 3, 4] (for a review see [5, 6]). This property, however, is not always easy to use, since typically one has to express the finite super Feynman diagrams in terms of ordinary (coordinate or momentum space) integrals which require regularization.

In this paper we show that it is possible to considerably simplify the expression of the supersymmetric massless ladder 4-point diagrams at arbitrary loop order, and rewrite them as 4-point conformal integrals. Interestingly we are able to carry out the whole calculation without introducing any regulator, despite the fact that individual component diagrams are divergent. Before going into details, let us briefly sketch our approach.

Consider a certain $N=1$ superdiagram, which by super power-counting is finite, for example the irreducible massless 4-point ladder diagram with four external scalar legs 1 depicted in Figure 1. Although the expression for the non supersymmetric massless $\varphi^{3}$ ladder with an arbitrary number of rungs was found long ago in [7], its generalization to the supersymmetric case is still not known.

To compute the ladder diagram in Figure 1 at a given order in perturbation theory, in coordinate space, one has to evaluate a multiple superspace integral of the form

$$
\begin{aligned}
G & \equiv G\left(x_{1}, x_{2}, x_{3}, x_{4}\right)= \\
& =\int \prod_{k} d^{4} y_{k} d^{2} \theta_{k} d^{2} \bar{\theta}_{k} \mathcal{W}\left(x_{1}, x_{2}, x_{3}, x_{4},\left\{y_{k}\right\},\left\{\theta_{k}\right\},\left\{\bar{\theta}_{k}\right\}\right),
\end{aligned}
$$

where $\mathcal{W}\left(x_{1}, x_{2}, x_{3}, x_{4},\left\{y_{k}\right\},\left\{\theta_{k}\right\},\left\{\bar{\theta}_{k}\right\}\right)$ denotes the product of super propagators, deriving from Wick contractions. Performing $\theta$ and $\bar{\theta}$ integrations in general gives as a result a linear combination of several component Feynman diagrams, so we can write eq. (1) in the form

$$
G=\int \prod_{k} d^{4} y_{k} \sum_{i} W_{i}\left(x_{1}, x_{2}, x_{3}, x_{4},\left\{y_{k}\right\}\right)
$$

where $W_{i}\left(x_{1}, x_{2}, x_{3}, x_{4},\left\{y_{k}\right\}\right)$ denote all allowed products of component field propagators (diagrams). The standard procedure for computing $G$ is to exchange in eq. (2) the order in which the sum over $i$ and the integration over

\footnotetext{
${ }^{1}$ In this paper we shall concentrate mostly on this case, but similar considerations hold also for other finite superdiagrams.
} 
$\left\{y_{k}\right\}$ are performed, obtaining

$$
\widetilde{G}=\sum_{i} \int \prod_{k} d^{4} y_{k} W_{i}\left(x_{1}, x_{2}, x_{3}, x_{4},\left\{y_{k}\right\}\right) .
$$

Note, however, that although by assumption the function $G$ is finite, some of the terms in $\widetilde{G}$ may diverge, so one has to introduce a regularization prescription to compute them. The regulator can be removed only after summing up all the terms.

This suggests that the necessity of regularization is not an intrinsic feature of finite correlation functions, but an artefact of our way of representing them in terms of ordinary component Feynman diagrams. Comparing eq. (21) and eq. (3) it is evident that the problem arises from exchanging the order of the integrations over $\left\{y_{k}\right\}$ and the summation over $i$. As we shall show, at least for the class of massless ladder 4-point superdiagrams, one can follow a different approach, which does not require any regulator at intermediate steps. To be explicit, we allow ourselves to exchange the order in which the integrations and the sum are performed in eq. (2) only as far as the result for each individual contribution to the sum remains finite. We then compute the integrals under the sum, perform the summation and then evaluate the remaining integrals. In formulae this corresponds to the following representation

$$
G=\int \prod_{k_{1}} d^{4} y_{k_{1}} \sum_{i} \int \prod_{k_{2}} d^{4} y_{k_{2}} W_{i}\left(x_{1}, x_{2}, x_{3}, x_{4},\left\{y_{k}\right\}\right),
$$

where each of the integrals

$$
\int \prod_{k_{2}} d^{4} y_{k_{2}} W_{i}\left(x_{1}, x_{2}, x_{3}, x_{4},\left\{y_{k}\right\}\right)
$$

is finite. The splitting of the integration points $\left\{y_{k}\right\}$ into two sets $\left\{y_{k_{1}}\right\}$ and $\left\{y_{k_{2}}\right\}$ depends on the diagram under consideration and, as we will show, in general is not unique. Different choices may give rise to apparently very different representations for the same correlation function $G$.

The paper is organized as follows. In Section 2 we set up the problem and provide a simplified expression of the 3-loop supersymmetric ladder diagram. In Section 3 we extend this result to all loop orders and express the massless supersymmetric ladder diagram with $L$ rungs in terms of conformal integrals. In Section 4 we derive two different diagrammatic representations for these conformal integrals. In Section 5, as a first step towards the evaluation of these integrals, we obtain an explicit expression for the 3-loop amplitude in the special case when all the points lie on a straight line and compute the singularities of the function. Finally, in Section 6 we give some conclusions. 


\section{The ladder super diagram}

We consider the massless Wess-Zumino model [1] with (Euclidean space) action

$$
\begin{aligned}
S & =\int d^{4} x\left\{\int d^{2} \theta d^{2} \bar{\theta} \Phi^{\dagger}(x, \theta, \bar{\theta}) \Phi(x, \theta, \bar{\theta})\right. \\
& \left.-\frac{g}{3 !} \int d^{2} \theta(\Phi(x, \theta, 0))^{3}-\frac{g}{3 !} \int d^{2} \bar{\theta}\left(\Phi^{\dagger}(x, 0, \bar{\theta})\right)^{3}\right\} .
\end{aligned}
$$

The chiral $N=1$ superfield $\Phi(x, \theta, \bar{\theta})$ contains a scalar $\varphi$, a Weyl fermion $\psi$ and an auxiliary filed $F$. The $\left\langle\Phi^{\dagger} \Phi\right\rangle$ propagator can be written in the compact form

$$
\left\langle\Phi^{\dagger}\left(x_{i}, \theta_{i}, \bar{\theta}_{i}\right) \Phi\left(x_{j}, \theta_{j}, \bar{\theta}_{j}\right)\right\rangle=\frac{1}{4 \pi^{2}} \mathrm{e}^{\left(\xi_{i i}+\xi_{j j}-2 \xi_{j i}\right) \cdot \partial_{j}} \frac{1}{x_{i j}^{2}},
$$

where $x_{i j}=x_{i}-x_{j}, \partial_{j}=\partial / \partial x^{j}$ and $\xi_{i j}^{\mu}=\theta_{i}^{\alpha} \sigma_{\alpha \dot{\beta}}^{\mu} \bar{\theta}_{j}^{\dot{\beta}}$. In this model only the 2-point function is divergent, while all higher point functions are finite. We remind that in the massless case the 3-point function may receive finite corrections [8, 9].

In this paper we shall concentrate on the irreducible massless ladder superdiagram with four external scalar legs $\varphi$ and $L$ rungs, which is depicted in Figure 1, namely

$$
G_{L}\left(x_{1}, x_{2}, x_{3}, x_{4}\right)=\left.\left\langle\varphi\left(x_{1}\right) \varphi^{\dagger}\left(x_{2}\right) \varphi^{\dagger}\left(x_{3}\right) \varphi\left(x_{4}\right)\right\rangle\right|_{g^{2 L}, \text { ladder }} .
$$

$G_{L}$ is proportional to $g^{2 L}$, so in the standard loop-order counting it is a $L$ loop diagram. Let us stress that with our choice for the external fields, the

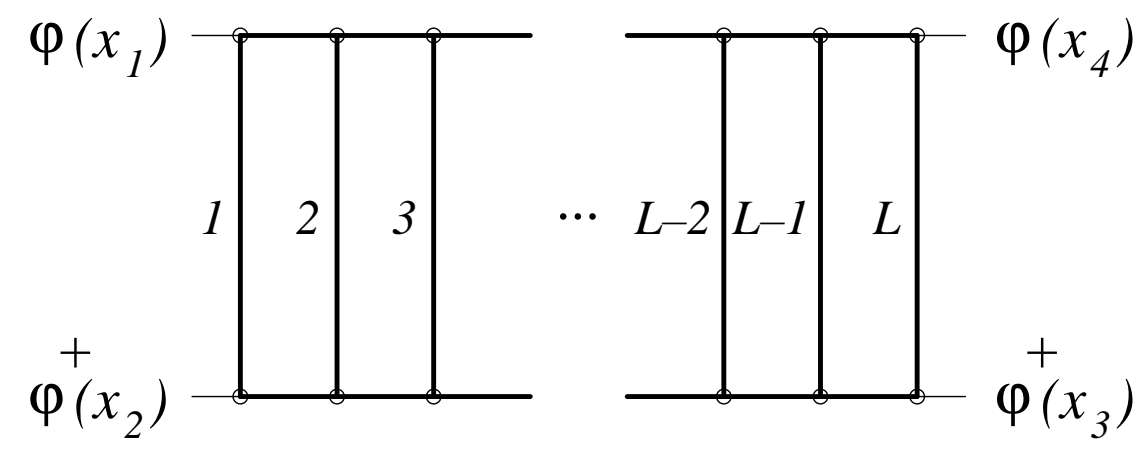

Figure 1: The ladder diagram with $L$ rungs.

ladder in Figure 1 is planar for odd values of $L$, while for even values of $L$ 
it is twisted. In Figure 1 all the internal lines represent superpropagators, hence effectively this single picture corresponds to a set of component field diagrams. In Figure 2 we present as an example the 6 component diagrams corresponding to the $L=3$ superdiagram.
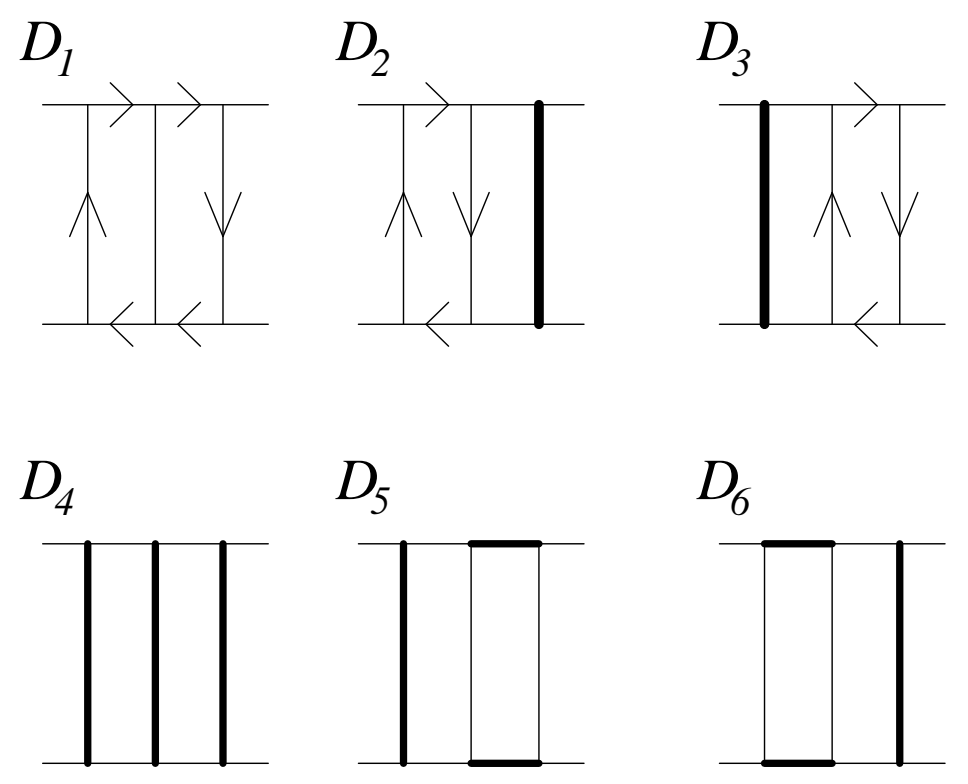

Figure 2: The six component diagrams for $L=3$.

Here and later in the paper for simplicity we shall suppress systematically all the factors $4 \pi^{2}$ in the propagators. Indeed, since $G_{L}$ involves $3 L+2$ propagators, the overall factor $1 /\left(4 \pi^{2}\right)^{3 L+2}$ can be reinstated at the end of the calculation. We use the following graphical notation for the component field propagators. We denote the $\varphi$ propagator, $1 / x^{2}$, by a thin continuous line, the fermion $\psi$ propagator, $\hat{x} / x^{4}=x_{\mu} \sigma^{\mu} / x^{4}$, by a thin continuous line with an arrow, and we represent by a fat line the $\delta$-function, corresponding to the propagator of the auxiliary field $F$. From the superpropagator of eq. (6) one can compute the relative sign factors weighting the different component diagrams in a superdiagram. It turns out that there is a $(-1)$ for each closed fermionic loop. Note also that with our graphical conventions there is a $(-1)$ sign for each $F$ propagator, since in Euclidean space the $F$ propagator is

$$
\square \frac{1}{x^{2}}=-4 \pi^{2} \delta(x) .
$$

With this conventions one finds that the expansion of the superdiagram in Figure 1, for $L=3$, in terms of the diagrams in Figure 2] is

$$
G_{3}\left(x_{1}, x_{2}, x_{3}, x_{4}\right)=-D_{1}+D_{2}+D_{3}-D_{4}-D_{5}-D_{6} .
$$


Note that while the superdiagram in Figure 1 is finite by $N=1$ super power counting, the integrals corresponding to the component diagrams in Figure 2 diverge. As we said, the standard procedure at this point is to introduce a regulator in order to make finite each of the component diagrams. We shall, instead, proceed differently and show that it is indeed possible to have all integrals finite at each step of the calculation, so that no regularization is actually necessary. We shall first illustrate the method on the example of $L=3$. In the next Section we shall extend it iteratively to general $L$.

Each of the diagrams in Figure 2 represents a 6 -fold integral over the internal integration points $y_{1}, \ldots, y_{6}$ (see Figure 3 ). Instead of trying to compute the 6-fold integral for each diagram and then summing the results, we split the integration into two 3-fold integrals: one set over integration variables with odd indices $\left\{y_{\text {odd }}\right\}$, and a second set over integration variables with even indices $\left\{y_{\text {even }}\right\}$ according to the chess-board like scheme of Figure 3 , As a result we can represent the 4-point function $G_{3}$ in the form

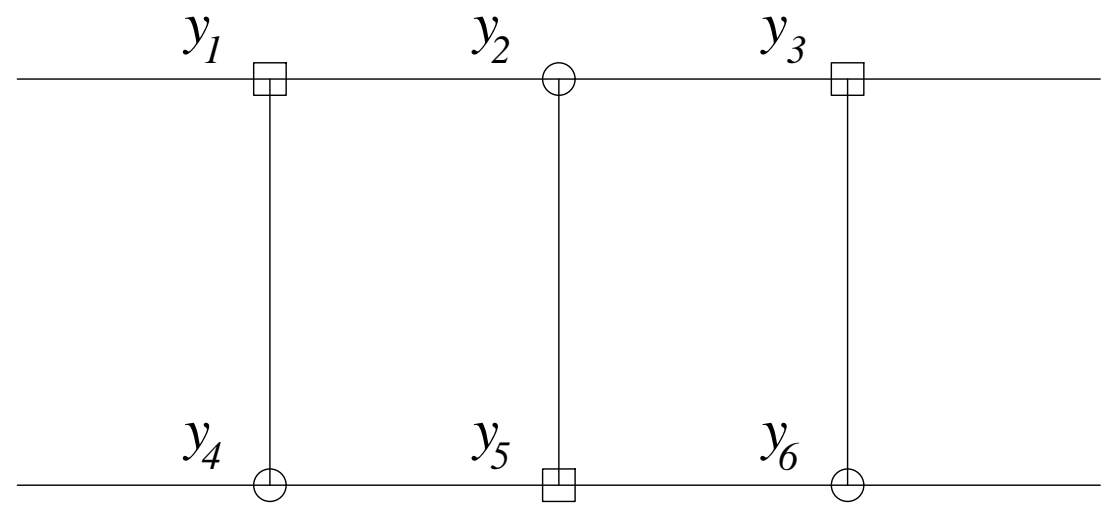

Figure 3: The two possible sets of integration points for $L=3$.

$$
G_{3}\left(x_{1}, x_{2}, x_{3}, x_{4}\right)=\int d^{4}\left\{y_{\text {even }}\right\} W_{3}\left(x_{1}, x_{2}, x_{3}, x_{4},\left\{y_{\text {even }}\right\}\right),
$$

where $W_{3}$ is given by an expression similar to eq. (9) where only integrations over $\left\{y_{\text {odd }}\right\}$ appear. We now show that all the integrations over $\left\{y_{\text {odd }}\right\}$ can be explicitly carried out in each of the diagrams $D_{1}, \ldots, D_{6}$ of Figure 2 and that they are all finite. Putting all the resulting terms together one obtains an expression for $W_{3}$, which can be integrated also over $\left\{y_{\text {even }}\right\}$ giving a finite result.

Note that one can exchange the order of the integrations over $\left\{y_{\text {even }}\right\}$ and over $\left\{y_{\text {odd }}\right\}$. This leads to two apparently very different representations for 
the same 4-point correlation function $G_{L}$ (see bellow). Their equivalence is a manifestation of the conformal invariance of $G_{L}$.

As can be noted by inspection, in each of the integration points in the diagrams of Figure 2 there is either a $\delta$-function coming from the propagator of the auxiliary field $F$, or two fermion propagators and a scalar propagator. The integration in the first case is trivial. To perform the integration in the second case we use the identity

$$
\widehat{\partial}_{1} \widehat{\partial}_{2} \int d^{4} x_{0} \frac{1}{x_{10}^{2} x_{20}^{2} x_{30}^{2}}=-4 \int d^{4} x_{0} \frac{\widehat{x_{10}} \widehat{x_{02}}}{x_{10}^{4} x_{20}^{4} x_{30}^{2}}=-4 \pi^{2} \frac{\widehat{x_{13}} \widehat{x_{32}}}{x_{13}^{2} x_{23}^{2} x_{12}^{2}},
$$

where $\widehat{x_{i j}}=\sigma_{\mu} x_{i j}^{\mu}$. This identity follows by applying the operator $\widehat{\partial}_{1} \widehat{\partial}_{2}$ to the explicit expression of the integral in the l.h.s. whose evaluation can be found in [11]. Eq. (11) is valid only in 4 space-time dimensions.

Due to the chess-board choice of $\left\{y_{\text {even }}\right\}$ and $\left\{y_{\text {odd }}\right\}$, the application of eq. (11) in any of the integration points does not alter the expressions involving the other integration points from the same set. The same is also true when a $\delta$-function from the $F$ propagator is involved. Hence, one can always perform the integrations over all the points belonging to one of the two sets. Choosing, for example, to integrate first over $\left\{y_{\text {odd }}\right\}$, we reduce the expression of $G_{3}$ to the form in eq. (10), where the function $W_{3}$ is a linear combination of products of propagators with numerators containing traces of products of (up to 6 ) $\widehat{x_{i j}}$ factors. Each term in $W_{3}$ separately contains high powers of $x^{2}$ in the denominator and diverges if integrated over $\left\{y_{\text {even }}\right\}$. However, after factoring out a common denominator and simplifying the numerator, it turns out that in the whole function, due to cancellations, there are only simple $1 / x^{2}$ poles and the remaining $\left\{y_{\text {even }}\right\}$ integrations display no divergencies. The final expression is

$$
\begin{aligned}
G_{3} & =-x_{14}^{2} \int d^{4} y_{2} d^{4} y_{4} d^{4} y_{6} W_{3}^{e}\left(x_{1}, x_{2}, x_{3}, x_{4} ; y_{2}, y_{4}, y_{6}\right) \\
& =-x_{23}^{2} \int d^{4} y_{1} d^{4} y_{3} d^{4} y_{5} W_{3}^{o}\left(x_{1}, x_{2}, x_{3}, x_{4} ; y_{1}, y_{3}, y_{5}\right)
\end{aligned}
$$

where the second identity follows by interchanging the order of the integrations over $\left\{y_{\text {even }}\right\}$ and $\left\{y_{\text {odd }}\right\}$. The functions $W_{3}^{e}$ and $W_{3}^{o}$ are given by the effective diagrammatic representation of Figure 4, where all the lines represent massless scalar propagators.

Since in each internal vertex in Figure 4 enter exactly 4 propagators, the integrals in eq. (12) are conformal, with conformal weights of the external legs equal to one. This means that the correlator can be expressed as a function of the two cross-ratio variables

$$
r=\frac{x_{13}^{2} x_{24}^{2}}{x_{12}^{2} x_{34}^{2}}, \quad s=\frac{x_{14}^{2} x_{23}^{2}}{x_{12}^{2} x_{34}^{2}}
$$



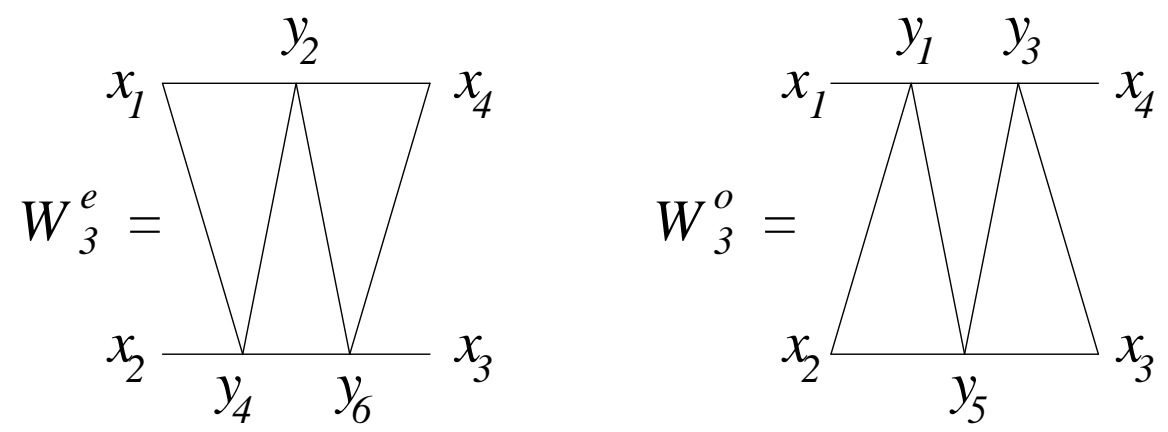

Figure 4: The two functions entering eq. (12) for the $L=3$ case.

in the form

$$
G_{3}\left(x_{1}, x_{2}, x_{3}, x_{4}\right)=\frac{1}{x_{12}^{2} x_{34}^{2}} F_{3}(r, s) .
$$

The integral in eq. (12) has been considered, in a particular kinematical regime appropriate for taking the on-shell limit, in [10] where also a MellinBarnes representation has been derived.

Before proceeding to the proof in the case of general $L$, we list the expressions for the simpler cases of $L=1$

$$
G_{1}\left(x_{1}, x_{2}, x_{3}, x_{4}\right)=-\int \frac{d^{4} x_{5}}{x_{15}^{2} x_{25}^{2} x_{35}^{2} x_{45}^{2}}=-\frac{\pi^{2}}{x_{12}^{2} x_{34}^{2}} \Phi^{(1)}(r, s),
$$

and $L=2$,

$$
\begin{aligned}
G_{2}\left(x_{1}, x_{2}, x_{3}, x_{4}\right) & =x_{14}^{2} \int \frac{d^{4} x_{5} d^{4} x_{6}}{x_{15}^{2} x_{16}^{2} x_{26}^{2} x_{35}^{2} x_{45}^{2} x_{46}^{2} x_{56}^{2}} \\
& =x_{23}^{2} \int \frac{d^{4} x_{5} d^{4} x_{6}}{x_{15}^{2} x_{25}^{2} x_{26}^{2} x_{35}^{2} x_{36}^{2} x_{46}^{2} x_{56}^{2}} \\
& =\frac{\pi^{4}}{x_{14}^{2} x_{23}^{2}} \Phi^{(2)}\left(\frac{r}{s}, \frac{1}{s}\right) .
\end{aligned}
$$

Interestingly, they both reduce to the massless $\varphi^{3}$ ladder, extensively studied in the literature [11, 7, 12]. The functions $\Phi^{(1)}$ and $\Phi^{(2)}$ are the first two members of an infinite family of conformal integrals introduced in [7]. The equivalence of the two representations for the $L=2$ case has been emphasized in [13] and used to prove the equality of apparently different conformal integrals. 


\section{All order result}

In principle one can proceed as discussed in the previous section also in the case of higher $L$ ladder diagrams. However, both the number of component diagrams as well as the complexity of each diagram grow very fast. Here we shall use a different approach, namely we shall derive recursively the general expression for the massless ladder superdiagram with four external scalar legs $\varphi$ and $L$ rungs. Expanding in component fields only the superpropagator in the leftmost rung one obtains a Bethe-Salpeter (BS) like recursive equation relating the scalar ladder with $L+1$ rungs $G_{L+1}$ to ladders with $L$ rungs, with 4 scalars $\left(G_{L}\right), 2$ fermions and 2 scalars $\left(G_{L}^{\psi}\right), 2$ auxiliary fields $F$ and 2 scalars $\left(G_{L}^{F}\right)$, as external legs. Precisely we get the relation

$$
\begin{aligned}
G_{L+1}\left(x_{1}, x_{2}, x_{3}, x_{4}\right)= & -\int d^{4} x_{5} d^{4} x_{6} \frac{\operatorname{tr}\left[\widehat{x}_{65} G_{L}^{\psi}\left(x_{5}, x_{6}, x_{3}, x_{4}\right)\right]}{x_{56}^{4} x_{16}^{2} x_{25}^{2}} \\
& -\int d^{4} x_{5} d^{4} x_{6} \frac{\delta\left(x_{56}\right)}{x_{16}^{2} x_{25}^{2}} G_{L}\left(x_{5}, x_{6}, x_{3}, x_{4}\right) \\
& +\int d^{4} x_{5} d^{4} x_{6} \frac{1}{x_{16}^{2} x_{25}^{2} x_{56}^{2}} G_{L}^{F}\left(x_{5}, x_{6}, x_{3}, x_{4}\right),
\end{aligned}
$$

where the ordering of the points corresponds to the convention of Figure 1 . The external fields in $x_{3}$ and $x_{4}$ are always scalars, the external fields in $x_{5}$ and $x_{6}$ are scalars in $G_{L}$, fermions in $G_{L}^{\psi}$ and auxiliary fields in $G_{L}^{F}$. The minus sign in the first term is due to the presence of an extra closed fermion loop, while the minus sign in the second term is due to the $(-1)$ sign in the $F$ propagator. After integrating the $\delta$-function in the second term and simplifying $G_{L}^{F}$, one obtains

$$
\begin{aligned}
G_{L+1}\left(x_{1}, x_{2}, x_{3}, x_{4}\right)= & -\int d^{4} x_{5} d^{4} x_{6} \frac{\operatorname{tr}\left[\widehat{x}_{65} G_{L}^{\psi}\left(x_{5}, x_{6}, x_{3}, x_{4}\right)\right]}{x_{56}^{4} x_{16}^{2} x_{25}^{2}} \\
& -\int d^{4} x_{5} \frac{1}{x_{15}^{2} x_{25}^{2}} G_{L}\left(x_{5}, x_{5}, x_{3}, x_{4}\right) \\
& +\int d^{4} x_{5} d^{4} x_{6} \frac{1}{x_{16}^{2} x_{25}^{2} x_{56}^{4}} G_{L-1}\left(x_{6}, x_{5}, x_{3}, x_{4}\right) .
\end{aligned}
$$

In this form the relation contains both $L$ and $L-1$ four scalar ladders, as well as the $L$-rung ladder diagram with two fermion and two scalar external lines. For the latter correlator, by expanding the leftmost rung, we derive 
the BS equation

$$
\begin{aligned}
G_{L+1}^{\psi}\left(x_{1}, x_{2}, x_{3}, x_{4}\right)= & \int d^{4} x_{5} d^{4} x_{6} \frac{\widehat{x}_{16} \widehat{x}_{65} \widehat{x}_{52}}{x_{16}^{4} x_{56}^{4} x_{25}^{4}} G_{L}\left(x_{5}, x_{6}, x_{3}, x_{4}\right) \\
& +\int d^{4} x_{5} d^{4} x_{6} \frac{\widehat{x}_{16} \widetilde{G}_{L}^{\psi}\left(x_{5}, x_{6}, x_{3}, x_{4}\right) \widehat{x}_{52}}{x_{16}^{4} x_{56}^{2} x_{25}^{4}},
\end{aligned}
$$

where $\widetilde{G_{L}^{\psi}}$ is obtained from $G_{L}^{\psi}$ by inverting the direction of the fermion line, which amounts to the substitution $\widehat{x}_{i j} \rightarrow \widehat{x}_{j i}, \widehat{x}_{i j} \widehat{x}_{j k} \widehat{x}_{k l} \rightarrow \widehat{x}_{l k} \widehat{x}_{k j} \widehat{x}_{j i}$ etc.

We shall first present the solutions of these equations, then we shall derive them. As already mentioned, the ladder superdiagram with $L$ rungs is planar for odd $L$, and twisted for even $L$. In order to work with only planar drawings we shall treat separately the two cases of even and odd $L$.

For the four scalars ladder and even values of $L$ we find

$$
\begin{aligned}
G_{L \text { even }}\left(x_{1}, x_{2}, x_{3}, x_{4}\right) & =x_{23}^{2} S_{1}^{e}\left(L ; x_{1}, x_{2}, x_{3}, x_{4}\right) \\
& =x_{14}^{2} S_{2}^{e}\left(L ; x_{1}, x_{2}, x_{3}, x_{4}\right) .
\end{aligned}
$$

The functions $S_{1}^{e}$ and $S_{2}^{e}$ are diagrammatically depicted in Figure 5, where all the lines represent massless scalar propagators, and the circles denote the $L$ integration points.
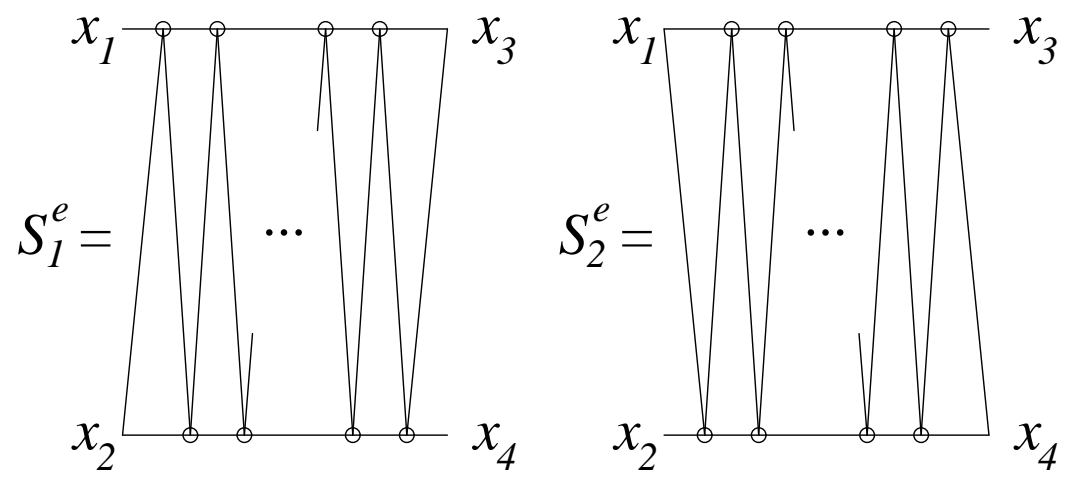

Figure 5: The case of even $L$.

For the four scalars ladder and odd values of $L$ we find

$$
\begin{aligned}
G_{L \text { odd }}\left(x_{1}, x_{2}, x_{3}, x_{4}\right) & =-x_{23}^{2} S_{1}^{o}\left(L ; x_{1}, x_{2}, x_{3}, x_{4}\right) \\
& =-x_{14}^{2} S_{2}^{o}\left(L ; x_{1}, x_{2}, x_{3}, x_{4}\right),
\end{aligned}
$$

where $S_{1}^{o}$ and $S_{2}^{o}$ are depicted in Figure 6 . 

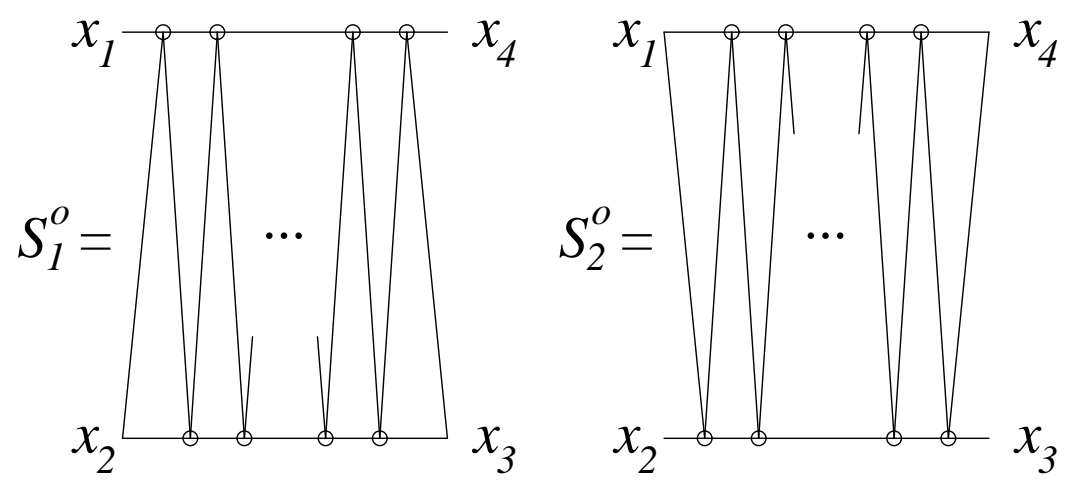

Figure 6: The case of odd $L$.

In the case of two fermions and two scalars in the external lines one finds for $L$ even

$$
G_{L \text { even }}^{\psi}\left(x_{1}, x_{2}, x_{3}, x_{4}\right)=-\int d^{4} x_{5} \frac{\widehat{x}_{14} \widehat{x}_{45} \widehat{x}_{52}}{x_{15}^{2} x_{25}^{4}} S_{1}^{o}\left(L-1 ; x_{1}, x_{5}, x_{4}, x_{3}\right),
$$

while for $L$ odd

$$
G_{L \text { odd }}^{\psi}\left(x_{1}, x_{2}, x_{3}, x_{4}\right)=\int d^{4} x_{5} \frac{\widehat{x}_{14} \widehat{x}_{45} \widehat{x}_{52}}{x_{15}^{2} x_{25}^{4}} S_{1}^{e}\left(L-1 ; x_{1}, x_{5}, x_{4}, x_{3}\right) .
$$

By a direct calculation one can check that the results for the $L=1$, $L=2$ and $L=3$ ladders are given by the expressions in eqs. (20) to (23). We now prove the general formulae by induction, using the BS equations (18) and (19). Assume that eqs. (20) to (23) hold up to a given $L$ and substitute them in the r.h.s of eq. (18) (we take for definiteness $L$ to be odd). With the help of eq. (11) we can perform one of the integrations in the term coming from $G_{L}^{\psi}$, simplify the trace of four $\sigma$ matrices in the numerator and use the identity

$$
\left(x_{i j} \cdot x_{k l}\right)=\frac{1}{2}\left(x_{i l}^{2}+x_{j k}^{2}-x_{i k}^{2}-x_{j l}^{2}\right)
$$

to express the resulting scalar products as squares of coordinate differences. Putting together all the terms, the potentially dangerous $1 / x_{56}^{4}$ behaviour in the last line of eq. (18) disappears, and we get the result in eq. (201). The other equations (21) to (23) are proven in a similar way.

The equality of the two different representations for $G_{L}$ in eq. (20) and eq. (21) is again a manifestation of the conformal invariance of these expressions. Actually, for any $L$, one can write

$$
G_{L}\left(x_{1}, x_{2}, x_{3}, x_{4}\right)=\frac{1}{x_{12}^{2} x_{34}^{2}} F_{L}(r, s),
$$


where $r$ and $s$ are the cross-ratios defined in eq. (13). This representation makes also manifest the invariance of the ladder diagram under the simultaneous exchanges $\left(x_{1} \leftrightarrow x_{4}\right.$ and $\left.x_{2} \leftrightarrow x_{3}\right) ;\left(x_{1} \leftrightarrow x_{3}\right.$ and $\left.x_{2} \leftrightarrow x_{4}\right) ;\left(x_{1} \leftrightarrow x_{2}\right.$ and $\left.x_{3} \leftrightarrow x_{4}\right)$.

\section{Alternative representations of $G_{L}$}

One can use the conformal invariance of the function $G_{L}$ eq. (25) to simplify the integral which has to be computed. In particular, without loss of generality one can perform a special conformal transformation to send one of the points (say $x_{4}$ ) to infinity. Setting

$$
f_{L}\left(x_{1}, x_{2}, x_{3}\right) \equiv \lim _{x_{4} \rightarrow \infty} x_{4}^{2} G_{L}\left(x_{1}, x_{2}, x_{3}, x_{4}\right)=\frac{1}{x_{12}^{2}} F_{L}\left(\frac{x_{13}^{2}}{x_{12}^{2}}, \frac{x_{23}^{2}}{x_{12}^{2}}\right)
$$

the calculation is reduced to that of an effective 3-point function. Indeed the $x_{4}$ dependence of $G_{L}$ can be reconstructed unambiguously from the knowledge of $f_{L}\left(x_{1}, x_{2}, x_{3}\right)$.

Taking the limit $x_{4} \rightarrow \infty$ has a simple graphical representation, namely it corresponds to erasing from the diagram all lines connecting $x_{4}$ with the other points. This explains how, combining the $x_{4} \rightarrow \infty$ limit with repeated application of the equality of the two rows of eqs. (20) and (21) (with the purpose of connecting as many as possible lines with the point $x_{4}$ ), we can reduce significantly the number of propagators in the diagram. Applying this procedure one can derive for $f_{L}\left(x_{1}, x_{2}, x_{3}\right)$ the representation depicted in Figure 7, where the left picture corresponds to $L$ even, and the right one to $L$ odd. The diagrams in Figure 7 are obtained by systematically replacing the representation involving $S_{1}^{e}$ with the one involving $S_{2}^{e}$ and the representation involving $S_{1}^{o}$ with the one involving $S_{2}^{o}$, for all subfunctions $G_{K}\left(x_{1}, x_{2}, y, x_{4}\right)$ for $K=L-1, L-2, \ldots, 2$, thus at each step increasing by one the number of propagators ending in $x_{4}$. Finally one sends $x_{4}$ to infinity.

Other expressions of the same function can be obtained either by using a different sequence of identities, or by sending some other point to infinity. The one we have singled out has two remarkable properties. On the one hand it involves the minimal possible number of propagators i.e. $2 L+1$, with an integrand having a numerator equal to one. On the other hand it has a form where exactly 3 propagators are attached to each integration point. Hence, the functions in Figure 7 are nothing but particular 3-point functions of composite operators in the massless $\varphi^{3}$ theory. Our analysis shows that these 3-point functions are finite. 

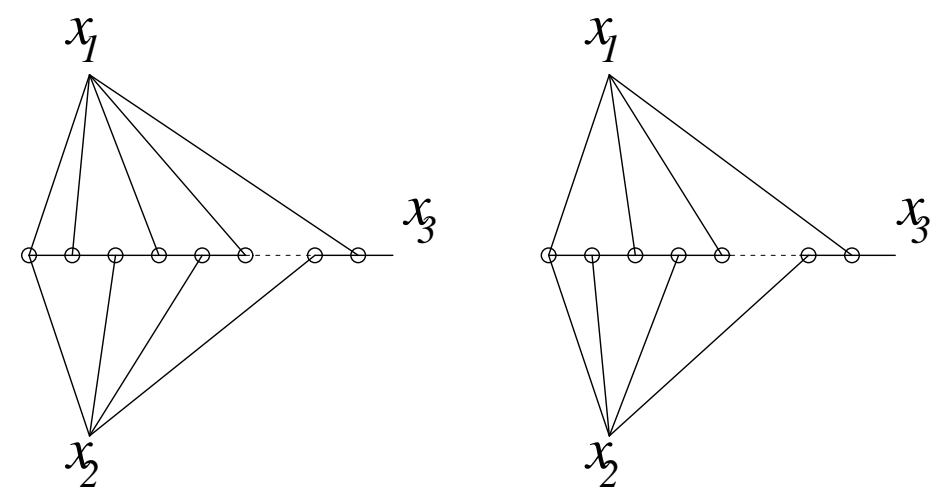

Figure 7: Representation for the 3-point function $f_{L}\left(x_{1}, x_{2}, x_{3}\right)$. The left picture corresponds to $L$ even, and the right one to $L$ odd. For $L$ odd there is an overall minus sign which is not shown in the picture.

In Figure 7 it is manifest that the functions $f_{L}\left(x_{1}, x_{2}, x_{3}\right)$ defined in eq. (26) satisfy a recursive relation, namely

$$
f_{L}\left(x_{1}, x_{2}, x_{3}\right)=-\int \frac{d^{4} x_{0}}{x_{10}^{2} x_{30}^{2}} f_{L-1}\left(x_{2}, x_{1}, x_{0}\right),
$$

and the differential equation

$$
\square_{x_{3}} f_{L}\left(x_{1}, x_{2}, x_{3}\right)=\frac{4 \pi^{2}}{x_{13}^{2}} f_{L-1}\left(x_{2}, x_{1}, x_{3}\right),
$$

which in turn implies a differential recursive relation for the functions $F_{L}(r, s)$.

One can also rewrite the integrals for $f_{L}$ as dual $L$-loop "momentum space like" integrals as shown in Figure 8. If one takes all the "momenta" to be incoming, then the one in the left vertex is $x_{12}$, the one in the right vertex is $x_{23}$, the one in the upper vertex is $x_{31}$, while those in the intermediate lines are constrained by "momentum" conservation holding in each vertex. Again the left picture corresponds to $L$ even, and the right one to $L$ odd. Similar representations can be derived also for the 4-point functions, (see e.g. [10] for the case $L=3$ ). Let us stress that, although all the propagators are massless, since in general $x_{i j}^{2} \neq 0$, one has to compute these "momentum space like" integrals with off-shell external legs.

Finally, we have to put back the overall power of $4 \pi^{2}$ which we suppressed for simplicity during the calculation. As already mentioned, the ladder diagram with $L$ rungs contains $3 L+2$ propagators, each proportional to $1 / 4 \pi^{2}$. On the other hand, in deriving eqs. (20) and (21) we have applied $L$ times the relation in eq. (11), producing a factor $\left(4 \pi^{2}\right)^{L}$. Hence, the overall multiplicative factor in $G_{L}$ and $f_{L}$ will be $1 /\left(4 \pi^{2}\right)^{2 L+2}$. 

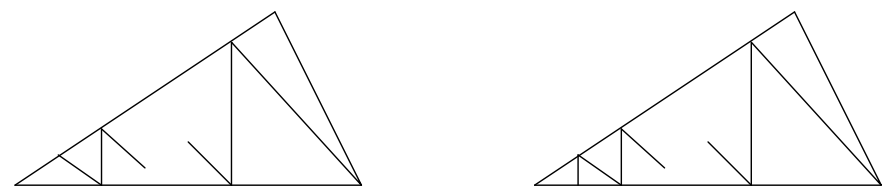

Figure 8: Dual representation for the 3-point function $f_{L}\left(x_{1}, x_{2}, x_{3}\right)$. The left picture corresponds to $L$ even, and the right one to $L$ odd.

\section{The 3-loop integral}

Note that the cross ratios $r$ and $s$ defined in eq. (13) cannot take arbitrary values. In particular, in the Euclidean regime they satisfy the constraints

$$
r \geq 0, \quad s \geq 0, \quad 1+r^{2}+s^{2}-4 r-4 s-4 r s \leq 0 .
$$

The last inequality is saturated when the 4 points $x_{1}, \ldots, x_{4}$ can be mapped by a conformal transformation to lie on a straight line, which, after sending by a special conformal transformation $x_{4}$ to infinity, reduces to the condition that $x_{1}, x_{2}$ and $x_{3}$ lie on a straight line. In this Section we shall compute the 3-loop integral

$$
I_{3}\left(x_{1}, x_{2}, x_{3}\right)=\int \frac{d^{4} x_{5} d^{4} x_{6} d^{4} x_{7}}{x_{15}^{2} x_{25}^{2} x_{26}^{2} x_{17}^{2} x_{37}^{2} x_{56}^{2} x_{67}^{2}}
$$

in this special kinematical regime 2 . The precise relation between the integral $I_{3}$ and the function $f_{3}$ is

$$
I_{3}\left(x_{1}, x_{2}, x_{3}\right)=-\left(4 \pi^{2}\right)^{8} f_{3}\left(x_{1}, x_{2}, x_{3}\right) .
$$

Note that, since $I_{3}\left(x_{1}, x_{2}, x_{3}\right)$ has singularities only at coinciding arguments, $x_{i}=x_{j}$, the knowledge of $I_{3}$ in this special configuration is sufficient to completely determine its singular behaviour. We use translation invariance to set $x_{2}=0$ and dilatation invariance to set $x_{3}=1$, with 1 a fixed unit vector. Since we have chosen the three points to lie on a line, the point $x_{1}$ can be parameterized as $x_{1}=u \mathbf{1}$. One has to treat separately the three cases, $u<0,0<u<1$ and $u>1$. We first consider $0<u<1$. In this representation the limit $u \rightarrow 0$ corresponds to $x_{12} \rightarrow 0$, while the limit $u \rightarrow 1$ corresponds to $x_{13} \rightarrow 0$. We shall analyze the limit $x_{23} \rightarrow 0$, which is related to $u \rightarrow \infty$, later.

\footnotetext{
${ }^{2}$ The result for the general case will be reported in a separate paper [14.
} 
In order to compute $I_{3}(u \mathbf{1}, 0, \mathbf{1})$, we use eq. (27) to relate $f_{3}$ to $f_{2}$, eqs. (26) and (16) to express $f_{2}$ in terms of $\Phi^{(2)}$ and the parametric integral representation [11]

$$
\Phi^{(2)}(x, y)=-\frac{1}{2} \int_{0}^{1} d t \frac{\ln (t)\left(\ln (t)+\ln \left(\frac{y}{x}\right)\right)\left(2 \ln (t)+\ln \left(\frac{y}{x}\right)\right)}{y t^{2}+(1-x-y) t+x} .
$$

Putting everything together, one gets

$$
\begin{aligned}
& I_{3}(u \mathbf{1}, 0, \mathbf{1})= \\
& =-\frac{\pi^{4}}{2} \int_{0}^{1} d t \int d^{4} x_{0} \frac{\ln (t)\left(\ln (t)+\ln \left(u^{2} / x_{0}^{2}\right)\right)\left(2 \ln (t)+\ln \left(u^{2} / x_{0}^{2}\right)\right)}{\left(x_{0}-\mathbf{1}\right)^{2}\left(x_{0}-u \mathbf{1}\right)^{2}\left(x_{0}-t u \mathbf{1}\right)^{2}} .
\end{aligned}
$$

In the integral over $x_{0}$ one can pass to spherical coordinates, such that $\left(x_{0} . \mathbf{1}\right)=R \cos (\theta)$ and perform first the integral over $\theta$ and then the radial integral in $R$. Taking into account also the trivial integration over the remaining two angles, the result is

$$
\begin{aligned}
& I_{3}(u \mathbf{1}, 0, \mathbf{1})=\frac{\pi^{6}}{u(1-u)} \times \\
& \left(2 \int_{0}^{1} \frac{d t \ln (t) \mathrm{Li}_{3}(t u)}{(1-t)}-3 \int_{0}^{1} \frac{d t \ln (t)^{2} \mathrm{Li}_{2}(t u)}{(1-t)}\right. \\
& -2 \int_{0}^{1} \frac{d t \ln (t)^{3} \ln (1-t u)}{(1-t)}-2 \ln (u) \int_{0}^{1} \frac{d t \ln (t) \mathrm{Li}_{2}(t u)}{(1-t)} \\
& -3 \ln (u) \int_{0}^{1} \frac{d t \ln (t)^{2} \ln (1-t u)}{(1-t)}-2 \ln (u)^{2} \int_{0}^{1} \frac{d t \ln (t) \ln (1-t u)}{(1-t)} \\
& +12 \mathrm{Li}_{4}(u)(\ln (1-u)-\ln (u)) \\
& -2 \mathrm{Li}_{3}(u)\left(\mathrm{Li}_{2}(u)+3 \ln (u)(\ln (1-u)-\ln (u))\right) \\
& +\mathrm{Li}_{2}(u) \ln (u)\left(2 \mathrm{Li}_{2}(u)-4 / 3 \ln (u)^{2}+2 \ln (u) \ln (1-u)\right) \\
& +\pi^{2} / 3\left(\mathrm{Li}_{3}(u)-\mathrm{Li}_{2}(u) \ln (u)-\ln (u)^{2} \ln (1-u)\right) \\
& \left.+2 \zeta(3)\left(\mathrm{Li}_{2}(u)+3 \ln (u) \ln (1-u)\right)-2 \pi^{4} / 15 \ln (1-u)\right)
\end{aligned}
$$

where $\operatorname{Li}_{n}(z)=\sum_{k=1}^{\infty} z^{k} / k^{n}$ are the index $n$ polylogarithms. One can rewrite eq. (34) in terms of harmonic polylogarithms [15] of weights up to 5. However, the supersymmetric massless ladder for $L \geq 3$, unlike the massless $\varphi^{3}$ ladder [7], cannot be expressed in a closed form in terms of usual polylogarithms $\mathrm{Li}_{n}$. 
From eq. (34) one can compute the leading singular behaviour of the function in the limit $u \rightarrow 0$ (corresponding to $x_{12} \rightarrow 0$ )

$$
\lim _{u \rightarrow 0} I_{3}(u \mathbf{1}, 0, \mathbf{1})=\left(-\frac{4}{3} \ln (u)^{3}+8 \ln (u)^{2}-20 \ln (u)+20-4 \zeta(3)\right) \pi^{6},
$$

and in the limit $u \rightarrow 1$ (corresponding to $x_{13} \rightarrow 0$ )

$$
\lim _{u \rightarrow 1} I_{3}(u \mathbf{1}, 0, \mathbf{1})=12 \zeta(3)(1-\ln (1-u)) \pi^{6} .
$$

To compute the limit $x_{23} \rightarrow 0$, we find it more convenient to use a different parametrization, namely to choose $x_{1}=\mathbf{1}$, with 1 a fixed unit vector and $x_{3}=$ $\tilde{u} \mathbf{1}$. For non-vanishing $u$ and $\tilde{u}$ the two parametrizations can be related by a scale transformation and the substitution $\tilde{u}=1 / u$. Using again eqs. (27), (26), (16) and (32) we get

$$
\begin{aligned}
& I_{3}(\mathbf{1}, 0, \tilde{u} \mathbf{1})= \\
& =-\frac{\pi^{4}}{2} \int_{0}^{1} d t \int d^{4} x_{0} \frac{\ln (t)\left(\ln (t)-\ln \left(x_{0}^{2}\right)\right)\left(2 \ln (t)-\ln \left(x_{0}^{2}\right)\right)}{\left(x_{0}-\mathbf{1}\right)^{2}\left(x_{0}-t \mathbf{1}\right)^{2}\left(x_{0}-\tilde{u} \mathbf{1}\right)^{2}} .
\end{aligned}
$$

In this expression one can take the limit $\tilde{u} \rightarrow 0$ under the integral, obtaining the finite value

$$
\lim _{\tilde{u} \rightarrow 0} I_{3}(\mathbf{1}, 0, \tilde{u} \mathbf{1})=20 \zeta(5) \pi^{6} .
$$

This is not a surprise, since in this limit the 4-point function $G_{3}$ reduces to a finite 3-point function. Actually this is true to all orders, hence all functions $G_{L}$ will be finite in the limit $x_{23} \rightarrow 0$. The finite 3-loop 3-point function gives rise to a logarithmic divergence in the 4-loop propagator. Our result, eq. (38), is in perfect agreement with the calculation of this propagator correction [16].

To summarize, the 3-loop integral $I_{3}\left(x_{1}, x_{2}, x_{3}\right)$ has a cubic logarithmic divergence for $x_{12} \rightarrow 0$, a linear logarithmic divergence for $x_{13} \rightarrow 0$ and has a finite limit for $x_{23} \rightarrow 0$.

\section{Conclusions and outlook}

In this paper we have worked out a method to substantially simplify the computation of the supersymmetric massless ladder 4-point diagrams with four external scalar legs at arbitrary loop order. The main result of our paper are eqs. (20) and (21) which express the ladder 4-point diagram with $L$ rungs, $G_{L}$, in terms of conformal integrals. For $L \geq 3$ the integrals in the supersymmetric case are different from the ones in the non supersymmetric 
case. We obtained two different representations for the conformal integrals in the supersymmetric case as 3-point functions and as dual momentum space like integrals. We derived the expression for the 3-loop integral $I_{3}\left(x_{1}, x_{2}, x_{3}\right)$ in the special case when the three points $x_{1}, x_{2}$ and $x_{3}$ lie on a straight line and we computed the singularities of the function. The resulting expression (see eq. (34)) turns out to be rather involved. For a generic configuration of $x_{1}, x_{2}$ and $x_{3}$ it is, not unexpectedly, even more complicated [14].

In principle, following the same steps as in Section 5 one can iterate the procedure and derive $f_{4}$ from $f_{3}$ etc.. However the complexity of the corresponding formulae grows very fast. Thus effectively such a direct approach seems very difficult, if not impossible, to pursue. We believe that a better understanding of the structure and the properties of the relevant conformal integrals is necessary at this point. Another interesting open problem is how to extend our construction to diagrams involving also vector superfields.

\section{Acknowledgements}

It is a pleasure to thank Emery Sokatchev for discussions. This work was supported in part by the INTAS grant 03-516346, MIUR-COFIN 2003-023852, NATO PST.CLG.978785, the RTN grants MRTNCT-2004-503369, EU MRTNCT-2004-512194, MRTN-CT-2004-005104. Partial support from MIUR-Italy (PRIN06) is also gratefully acknowledged.

\section{References}

[1] J. Wess and B. Zumino, "A lagrangian model invariant under supergauge transformations", Phys. Lett. B 49 (1974) 52.

[2] J. Iliopoulos and B. Zumino, "Broken supergauge symmetry and renormalization", Nucl. Phys. B 76 (1974) 310.

[3] S. Ferrara, J. Iliopoulos and B. Zumino, "Supergauge invariance and the Gell-Mann Low eigenvalue", Nucl. Phys. B 77 (1974) 41.

[4] M. T. Grisaru, W. Siegel and M. Rocek, "Improved Methods For Supergraphs", Nucl. Phys. B 159 (1979) 429.

[5] S. J. Gates, M. T. Grisaru, M. Rocek and W. Siegel, "Superspace, or one thousand and one lessons in supersymmetry", Front. Phys. 58 (1983) 1 arXiv:hep-th/0108200. 
[6] P. West, "Introduction to supersymmetry and supergravity", World Scientific, (1990), see Chapter 17.

[7] N. I. Usyukina and A. I. Davydychev, "Exact results for three and four point ladder diagrams with an arbitrary number of rungs", Phys. Lett. B 305 (1993) 136.

[8] P. C. West, "A Comment on the nonrenormalization theorem in supersymmetric theories", Phys. Lett. B 258 (1991) 375.

[9] I. Jack, D. R. T. Jones and P. C. West, "Not the no - renormalization theorem?", Phys. Lett. B 258 (1991) 382.

[10] D. Nguyen, M. Spradlin and A. Volovich, "New Dual Conformally Invariant Off-Shell Integrals", Phys. Rev. D 77 (2008) 025018 arXiv:0709.4665 [hep-th]].

[11] N. I. Usyukina and A. I. Davydychev, "An Approach to the evaluation of three and four point ladder diagrams", Phys. Lett. B 298 (1993) 363.

[12] D. J. Broadhurst, "Summation of an infinite series of ladder diagrams", Phys. Lett. B 307 (1993) 132.

[13] J. M. Drummond, J. Henn, V. A. Smirnov and E. Sokatchev, "Magic identities for conformal four-point integrals", JHEP 0701 (2007) 064 arXiv:hep-th/0607160.

[14] G. C. Rossi and Ya. S. Stanev, in preparation.

[15] E. Remiddi and J. A. M. Vermaseren, "Harmonic polylogarithms", Int. J. Mod. Phys. A 15 (2000) 725 arXiv:hep-ph/9905237].

[16] D. I. Kazakov, "Calculation Of Feynman Integrals By The Method Of 'Uniqueness' ", Theor. Math. Phys. 58 (1984) 223 [Teor. Mat. Fiz. 58 (1984) 343]; "The Method Of Uniqueness, A New Powerful Technique For Multiloop Calculations", Phys. Lett. B 133 (1983) 406. 